\title{
Konservierungsmittel in Impfstoffen - die Situation in der Schweiz
}

\author{
A. Stalder
}

Ursprünglich wurden Konservierungsmittel in Impfstoffen eingesetzt, um die Sterilität bei der Herstellung und - im Falle von Mehrdosenbehältern - bei der Entnahme zu garantieren. Die verbesserten Herstellungsbedingungen und der Einsatz von Monodosen erlauben es, heute mehr und mehr auf Konservierungsmittel zu verzichten. Diese Übersichtsarbeit soll aufzeigen, welche Konservierungsmittel in Impfstoffen für den Schweizer Markt zum Einsatz kommen und wie es um deren Sicherheit steht. Die organische Quecksilberverbindung Thiomersal, welche unter Verdacht steht, das Gehirn von Neugeborenen und Säuglingen zu schädigen und an der Auslösung von Autismus beteiligt zu sein, wurde mehrheitlich durch 2-Phenoxyethanol ersetzt oder gänzlich weggelassen. Heute können in der Schweiz alle Kinderimpfungen mit thiomersalfreien Einzeldosen durchgeführt werden. Da allerdings Langzeitsicherheitsdaten für 2-Phenoxyethanol nach parenteraler Anwendung erst vereinzelt vorliegen, begrüsst Swissmedic den gänzlichen Verzicht auf Konservierungsmittel in Einzeldosen bei kontinuierlicher Verbesserung der Herstellprozesse, wobei im Sinne einer Nutzen-Schaden-Analyse der Einhaltung der Sterilität gegenüber dem gänzlichen Verzicht auf ein Konservierungsmittel oberste Priorität zukommen soll. So müssen z.B. Mehrfachdosen, wie sie insbesondere durch die WHO in Entwicklungsländern eingesetzt werden, auf jeden Fall weiterhin konserviert werden.

\section{Einleitung}

Konservierungsmittel können inaktivierten Impfstoffen beigefügt werden, um den schädlichen Auswirkungen allfälliger Kontaminationen mit Bakterien und Pilzen entgegenzuwirken. Lebendimpfstoffe (z.B. Masern, Mumps, Röteln, Gelbfieber, Tuberkulose) können nicht konserviert werden, da die attenuierten Viren und Bakterien so geschädigt würden, dass die Impfstoffwirkung nicht mehr garantiert wäre. Für inaktivierte Impfstoffe in Mehrdosenbehältern ist ein Konservierungsmittelzusatz zwingend vorgeschrieben, da es in der Vergangenheit zu Todesfällen infolge Kontamination bei Mehrfachentnahmen kam [1]. Dieses Risiko kann jedoch nicht vollständig eliminiert werden, und es sind Fälle in der Literatur erwähnt, wo Mehrdosenbehälter trotz Konservierung bakteriell verunreinigt wurden $[2,3]$. In der Schweiz sind alle Impfstoffe als Einzeldosen erhältlich.

Die meistverwendeten Konservierungsmittel in Impfstoffen sind Thiomersal, 2-Phenoxyethanol und Phenol. Infolge des möglichen $\mathrm{Zu}-$ sammenhangs mit Autismus und der bekannten Toxizität und schlechter Umweltverträglichkeit der Thiomersalkomponente Quecksilber, haben im Sinne einer Risikominimierung sowohl Schweizer, europäische, wie US-Behörden bereits vor Jahren Empfehlungen abgegeben, thiomersalfreien Impfstoffen den Vorzug zu geben. Heute sind alle Kinderimpfungen gemäss Schweizer Impfplan [4] in thiomersalfreien Einzeldosen erhältlich. Trotzdem ist das Thema «Thiomersal als möglicher Auslöser von Autismus» nicht abgeschlossen und nach wie vor Gegenstand von Diskussionen in der Öffentlichkeit, in den Medien und im Parlament. Die Zahl der Autismusfälle ist weiterhin steigend und neurologische Störungen können sich verzögert entwickeln. Die Ursache(n) von Autismus ist/ sind nicht geklärt. Besorgte Eltern von Kindern, die noch mit thiomersalhaltigen Impfstoffen geimpft wurden, stellen Fragen bezüglich Spätfolgen. Nach wie vor werden weltweit viele thiomersalhaltige Impfstoffe in Mehrfachdosen eingesetzt. Dies insbesondere in Drittweltländern, die sich Kampagnen mit Einzeldosen nicht leisten können, aber auch z.B. in den USA, wo momentan die Abfüllkapazität unzureichend ist, um rechtzeitig vor der Grippesaison genügend Influenzaimpfstoffmonodosen für die Bevölkerung bereitzustellen.

Nachfolgend sollen Fragen rund um Konservierungsmittel in Impfstoffen beantwortet und aus Sicht der Behörden die Situation in der Schweiz dargestellt werden.

\section{Welche Konservierungsmittel kommen in für den Schweizer Markt zugelassenen Impfstoffen zum Einsatz, und in welchen Mengen?}

Die Konservierungsmittel, welche in durch Swissmedic für den Schweizer Markt zugelassenen Impfstoffen zum Einsatz kommen, sind Thiomersal, 2-Phenoxyethanol und Phenol. Thiomersal ist bei Zugabe zum Endprodukt in Mengen von 25 bis $80 \mu$ g pro Dosis vorhanden. Wird Thiomersal nicht zur Konservierung des fertigen Impfstoffs, sondern zur Verhinderung von Keimwachstum während des Herstellprozesses verwendet, kann das Impfstoffendprodukt 
Thiomersalrückstände von maximal 2,5 $\mu \mathrm{g}$ pro Dosis enthalten. Tabelle 1 führt die von Swissmedic zugelassenen thiomersalhaltigen Impfstoffe auf, mit Angabe der zugesetzten Menge. Bei den Impfstoffen gegen Tetanus, beim kombinierten Diphtherie-Tetanus-Impfstoff und den Impfstoffen gegen Hepatitis A, B und Influenza stehen thiomersalfreie Alternativen zur Verfügung. Einzig für den Impfstoff gegen Leptospiren (Spirolept ist indiziert ab dem vollendeten 18. Lebensjahr) liegt keine thiomersalfreie Alternative vor. Da seitens der zuständigen Behörde (bis 31. Dezember 2001 das Bundesamt für Gesundheit, seit 1. Januar 2002 Swissmedic) schon frühzeitig Anstrengungen unternommen wurden, die Firmen $\mathrm{zu}$ motivieren, thiomersalfreie Impfstoffe in Einzeldosen herzustellen, können heute alle Impfungen gemäss Schweizer Impfplan thiomersalfrei durchgeführt werden. 2-Phenoxyethanol (2-PE) wird typischerweise in Mengen von 2,5 bis $5 \mathrm{mg}$ pro Impfstoffdosis eingesetzt. Im Vergleich mit Thiomersal kommt 2-PE somit in 50- bis 100fach höheren Konzentrationen zum Einsatz. Tabelle 2 zeigt die von Swissmedic zugelassenen 2-PE-haltigen Impfstoffe mit Angabe der zugesetzten Menge. Die Mengen von Phenol in den Impfstoffendprodukten bewegen sich zwischen 0,2 und 1,25 mg pro Dosis. Tabelle 3 listet die von Swissmedic zugelassenen Impfstoffe mit Phenol als Konservierungsmittel auf mit Angabe der zugesetzten Menge. Da Konservierungsmittel antimikrobiell wirksam sind und somit auch toxisches Potential für den Menschen haben, sollen im folgenden die Eigenschaften der verschiedenen Konservierungsmittel diskutiert werden.

\section{Was ist Thiomersal und wie sicher ist die Substanz?}

Thiomersal (Abb. 1) ist eine organische Quecksilberverbindung (49,6\% des Gewichtes entsprechen reinem Quecksilber), die seit den 1930er Jahren zur Konservierung von inaktivierten Impfstoffen verwendet wird. Thiomersal wird üblicherweise in Konzentrationen von 0,005\% bis $0,01 \%$ eingesetzt. Neben Impfstoffen werden auch Augen- und Nasentropfen, Immunglobuline, Kontaktlinsenflüssigkeiten und Kosmetika gelegentlich mit Thiomersal konserviert. Andere Bezeichnungen für Thiomersal sind: Thimerosal, Merthiolat, Quecksilbernatriumethylthiosalizylat oder Natriumtimerfonat. Die antimikrobielle Wirkung ist auf die Ethylquecksilberkomponente zurückzuführen, die nach spontaner oder enzymatischer Spaltung in Ethylquecksilber und
Thiosalizylat freigesetzt wird. Thiomersal wirkt bakterizid bei saurem und bakteriostatisch und fungistatisch bei neutralem und alkalischem pH-Wert. Gegen Sporenbildner und Viren zeigt Thiomersal keine Wirkung. Thiomersal ist das weltweit am häufigsten gebrauchte Konservierungsmittel und wird, auch historisch bedingt, vor allem bei Toxoidimpfstoffen (Diphtherie, Tetanus) eingesetzt. Thiomersal beeinträchtigt die Wirksamkeit von inaktivierten Poliomyelitisimpfstoffen (IPV) [5, 6] und kann deshalb auch in Kombinationsimpfstoffen, welche IPV enthalten, nicht verwendet werden.

\section{Akute Toxizität}

Die meisten Nebenwirkungen, die nach dem Einsatz von thiomersalhaltigen Impfstoffen, aber auch nach dem Gebrauch von Augentropfen und Kontaktlinsenflüssigkeiten auftreten, sind lokale allergische Typ-IV-Sensibilisierungen $^{a}$. Bei Impfstoffgabe kann es dadurch lokal zu Rötung, Schmerz, Verhärtung und/oder Schwellung kommen [7]. Vereinzelt treten auch Urtikaria oder generalisiertes Ekzem auf. Thiomersal am Auge kann Rötung, Irritation bis hin zu allergischer Bindehautentzündung hervorrufen. Thiomersal hat ein stark sensibilisierendes Potential. Es wird postuliert, dass die Thiosalizylatkomponente für die allergische Reaktion verantwortlich ist [8]. Thiomersal in höheren Dosen ist schädlich nach Inhalation, Kontakt mit der Haut und peroraler Einnahme. Tierexperimentell wurden neoplastische, teratogene und fruchtbarkeitsschädigende Wirkungen beobachtet.

Akute Toxizitätsdaten für Thiomersal sind in Tabelle 4 aufgeführt. Zum Zeitpunkt der Einführung von Thiomersal als Konservierungsmittel lagen Daten aus Studien an Tieren und Menschen vor, die auf einen sicheren und wirksamen Einsatz schliessen liessen. So wurden Dosen von $20 \mathrm{mg} / \mathrm{kg}$ beim Kaninchen und $45 \mathrm{mg} / \mathrm{kg}$ bei der Ratte toleriert. Die i.v. Gabe an 22 Personen mit Dosen von bis zu $26 \mathrm{mg} / \mathrm{kg}$ zeigte keine toxische

\section{Abbildung 1}

Strukturformel von Thiomersal.

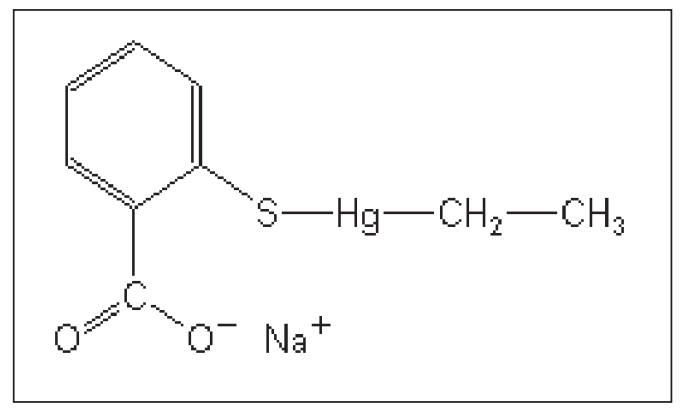




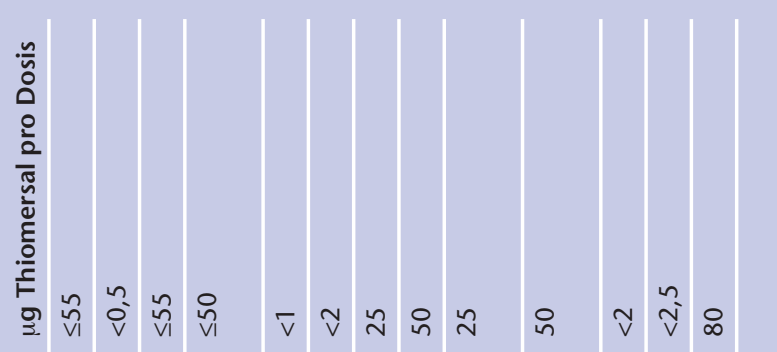

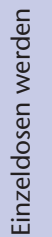
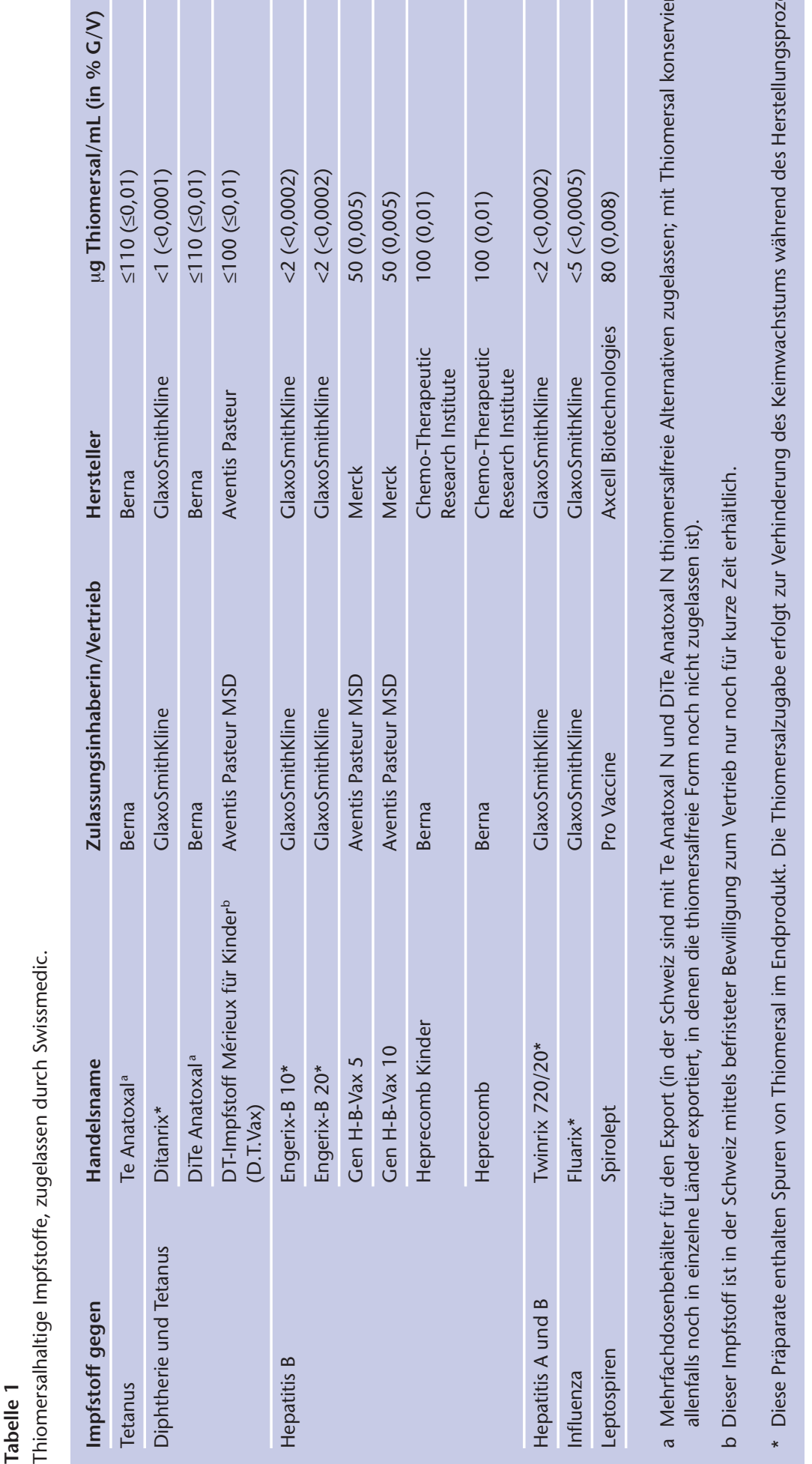

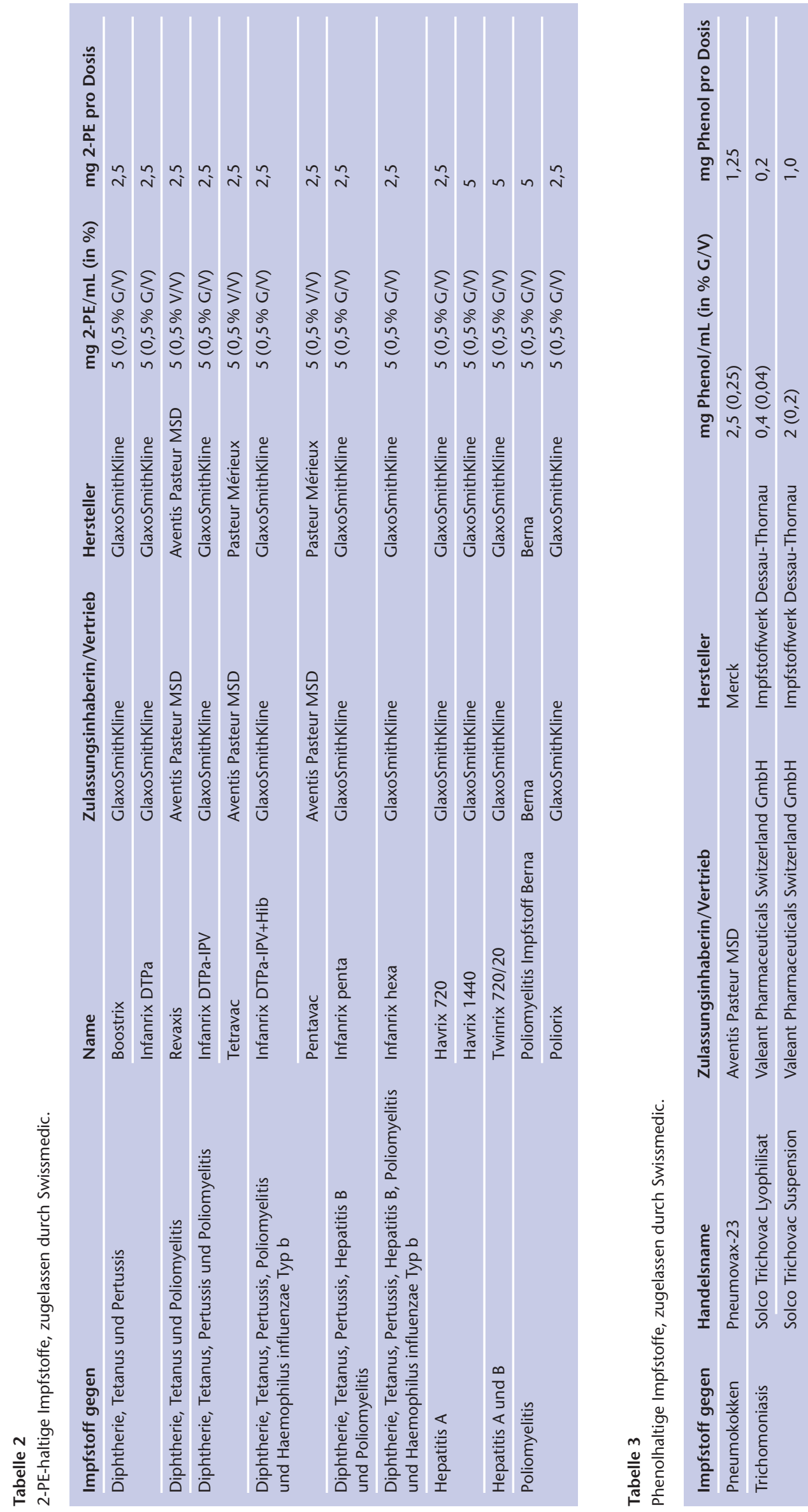
Tabelle 4

Akute Toxizitätsdaten (LD50 in mg/kg) für Thiomersal, 2-Phenoxyethanol und Phenol.

\begin{tabular}{llccc} 
Spezies & Route & Thiomersal & 2-Phenoxyethanol & Phenol \\
Maus & Oral & 91 & 933 & 300 \\
& Intravenös & 30 & - & 110 \\
\hline Ratte & Intraperitoneal & 54 & - & - \\
& Oral & 75 & 1260 & 320 \\
& Intraperitoneal & - & 554 & - \\
& Subkutan & 98 & - & 460 \\
& Dermal & - & 14422 & 670 \\
\hline Kaninchen & Dermal & - & 5000 & 850
\end{tabular}

Wirkung, wobei jedoch 7 der 22 Probanden nur während eines Tages beobachtet wurden [9]. Dem stehen Berichte von Quecksilbervergiftungen nach Gabe von Präparaten mit Thiomersalmengen von 3 bis einigen $100 \mathrm{mg} / \mathrm{kg}$ gegenüber. So wurde z. B. irrtümlich eine Chloramphenikollösung mit dem 1000fachen der notwendigen Menge Thiomersal intramuskulär vier Kindern und zwei Erwachsenen gegeben. Bei allen sechs Individuen kam es zu Vergiftungen und fünf davon starben. Ein sechs Wochen alter Säugling (5 kg), der überlebte, erhielt $450 \mathrm{mg}$ Thiomersal (90 mg/kg). Die fünf Personen, die starben, erhielten zwischen 1,0 und 16,5 g Thiomersal, was Mengen von 71, 133, 188, 324 und $330 \mathrm{mg}$ Thiomersal pro kg Körpergewicht entspricht [10]. Die tiefste publizierte tödliche Dosis liegt bei $60 \mathrm{mg} / \mathrm{kg}$ nach intermittierender, intraauraler Verabreichung über 4 Wochen an ein Kind [11].

\section{Aufnahme, Verteilung, Metabolisierung und Elimination}

Organische Quecksilberverbindungen werden peroral leichter absorbiert als anorganische Formen von Quecksilber, die mehrheitlich mit den Faeces wieder ausgeschieden werden. Im Körper wird Thiomersal enzymatisch oder spontan in Thiosalizylat und Ethylquecksilber gespalten. Ethylquecksilber bindet an Blutzellen und andere Gewebe. Organische Alkylquecksilberverbindungen (z.B. Ethyl- und Methylquecksilber ${ }^{\text {b) }}$ verteilen sich im ganzen Körper, wobei hohe Konzentrationen im Gehirn auftreten können. Die Elimination erfolgt primär über Galle und Stuhl, hauptsächlich in Form von anorganischem Quecksilber. In einer Studie erhielten 40 Säuglinge ( $\leq 6$ Monate) thiomersalhaltige $\mathrm{DTaP}^{c}$, Hepatitis-B- und teilweise auch Hib ${ }^{\mathrm{d}}$-Impfstoffe. 21 Säuglinge erhielten thiomersalfreie Impfstoffe und dienten als Kontrolle. 3 bis 28 Tage nach der Impfung wurden Blut-, Urin- und Stuhlproben genommen und auf ihren Quecksilbergehalt überprüft. Für Ethylquecksilber wurde eine Halbwertszeit von 7 Tagen gefunden (im Vergleich zu 40 bis 50 Tagen für Methylquecksilber, das vorwiegend in Lebensmitteln vorkommt) [12]. Organische Quecksilberverbindungen passieren die Plazenta und gehen auch in die Muttermilch über [13]. Das US National Institute of Allergy and Infectious Diseases (NIAID) unterstützt Studien, die den Metabolismus von Thiomersal untersuchen. So läuft u.a. eine Studie in Argentinien, in der Quecksilberblutspiegel und Quecksilberexkretion bei Kindern bestimmt werden, die thiomersalhaltige Grundimmunisierungsimpfungen erhalten. Diese Studie sollte im Frühling 2005 beendet werden. Eine weitere Studie, die ebenfalls in Argentinien stattfinden wird, soll auch Frühgeburten (32. bis 37. Woche) und Neugeborene mit geringem Geburtsgewicht von 2 bis $3 \mathrm{~kg}$ einschliessen. Diese Studie wird voraussichtlich nicht vor 2007 beendet sein.

\section{Chronische Toxizität}

Da Quecksilber in Gewässern, Luft, Boden, Pflanzen und Tieren (z.B. in Meerfischen) weit verbreitet ist, kann sich der Mensch einer Exposition nicht vollständig entziehen. In der Allgemeinbevölkerung liegt die Quecksilberbelastung bei durchschnittlich $7 \mu \mathrm{g} / \mathrm{Tag}$ (ohne Amalgam) [14].

Grenzwerte für die Quecksilberaufnahme basieren auf epidemiologischen Studien und Laborexperimenten mit Methylquecksilber. Die Limiten liegen je nach Behörde zwischen 0,1 $\mu \mathrm{g}$ pro kg Körpergewicht (KG) pro Tag (US Environmental Protection Agency EPA [15]) und 0,47 $\mu \mathrm{g}$ pro kg KG pro Tag (WHO [16]). Da Grenzwerte für Ethylquecksilber fehlen, wurden diejenigen für Methylquecksilber auch für Risikoabschätzungen zu Thiomersal angewendet. Bei der Verabreichung eines thiomersalhaltigen Impfstoffes (25 bis $80 \mu \mathrm{g}$ Thiomersal pro Dosis, Tab. 1) an einen Säugling von $5 \mathrm{~kg}$ würde somit die $0,1-\mu g$ Quecksilbertageslimite um das 25- bis 80fache überschritten.

Zwecks Risikominimierung haben die Zulassungsbehörden in Europa, den USA und der Schweiz die Impfstoffhersteller angehalten, die Entwicklung von Impfstoffen mit alternativen Konservierungsmitteln (z.B. 2-Phenoxyethanol) voranzutreiben oder die Konservierungsmittel ganz wegzulassen [17-21]. Das US National Institute of Allergy and Infectious Diseases (NIAID) unterstützt zusammen mit dem US National Institute of Environmental Health Sciences (NIEHS) eine Studie an Primaten, in der Pharmakokinetik und Gewebeverteilung von Thiomersal und Methylquecksilber verglichen werden. 
Diese Studie soll Klarheit darüber geben, ob Richtlinien für Methylquecksilber tatsächlich auch zu Risikoabschätzungen für Thiomersal angewendet werden können. Erste Resultate zeigen, dass Quecksilber sowohl in Form von Methylquecksilber (orale Einnahme) als auch und in Form von Thiomersal (Ethylquecksilber, intramuskuläre Gabe) rasch absorbiert und in Blut und Gehirn verteilt wird. Die gemessenen Quecksilberkonzentrationen nach Thiomersalgabe in Blut und Gehirn waren tiefer und nahmen rascher ab als diejenigen nach Methylquecksilbergabe. Wöchentliche Gaben von Methylquecksilber führten zu deutlicher, wöchentliche Gaben von Thiomersal dagegen nur zu schwacher Quecksilberakkumulation im Blut.

\section{Vergiftungen}

Der Toxizität von Methylquecksilber wurde man sich erstmals bewusst durch Massenvergiftungen nach Fischkonsum in der japanischen Minimata-Bucht zwischen 1953 und 1969 [22]. 121 Personen starben, darunter 12 Kinder, und über 1200 Menschen erkrankten schwer. Methylquecksilberhaltiges Abwasser einer Kunststofffabrik war der Grund für die Vergiftungen. In den Organen der Verstorbenen wurden folgende Quecksilbermengen gefunden: Leber: 22,0 bis $70,5 \mathrm{mg} / \mathrm{kg}$, Gehirn: 2,6 bis 24,8 mg/kg, Niere: 21,2 bis $140,0 \mathrm{mg} / \mathrm{kg}$.

1970 kam es im Irak zu einer Massenvergiftung nach Verzehr von Saatgut, das mit einem methylquecksilberhaltigen Fungizid behandelt worden war [23]. Dabei erkrankten 6530 Personen, wovon 459 starben. Die eingenommenen Quecksilbermengen lagen zwischen total 50 und $400 \mathrm{mg}$. Bei diesen Vergiftungen zeigte sich, dass ungeborene Kinder viel empfindlicher reagieren als Erwachsene. So kam es u.a. zu zerebralen Lähmungen und geistiger Behinderung. Methylquecksilber ist neurotoxisch.

\section{Besteht ein Zusammenhang zwischen Thiomersal und neurologischen Störungen, insbesondere Autismus, bei Kindern?}

Vorwiegend in den USA, aber auch in Europa und der Schweiz haben in den letzten Jahren die Autismusfälle bei Kindern zugenommen. Statistische Auswertungen in Kalifornien haben ergeben, dass zwischen 1987 und 2002 die Anzahl autistischer Kinder um 634 Prozent auf 20377 gestiegen ist [24]. Eine analoge, wenn auch nicht so ausgeprägte Zunahme der Fälle von Autismus beobachtet man auch in Europa. In der Schweiz haben gemäss Bundesamt für Sozialversicherung die gemeldeten Autismusfälle zwischen 1993 und 2003 bei Jungen um 91\% und bei Mädchen um $67 \%$ zugenommen.

Die starke Zunahme des Autismus, insbesondere in den USA, kann noch nicht befriedigend erklärt werden. Da in der beschriebenen Zeitspanne auch die Anzahl thiomersalhaltiger Impfungen, die amerikanische Kinder infolge erweiterter staatlicher Empfehlungen erhielten, stark angestiegen ist, wurde ein Zusammenhang zwischen Thiomersal und Autismus vermutet. Säuglinge erhielten in den USA 1999 und während der Grundimmunisierung in den ersten sechs Lebensmonaten kumulativ bis zu $200 \mu \mathrm{g}$ Quecksilber [25]. Dieser Wert liegt deutlich über dem Grenzwert der EPA von 0,1 $\mu$ g pro kg Körpergewicht (KG) pro Tag, wie Tab. 5 zeigt. Auch in der Schweiz konnte es in der Vergangenheit zu Grenzwertüberschreitungen kommen.

2001 kam das US Immunization Safety Committee of the National Academy of Science's Institute of Medicine (IOM) zum Schluss, dass ein kausaler Zusammenhang zwischen Thiomersal und untersuchten neurologischen Störungen wie Autismus, Aufmerksamkeitsdefizitsyndrom (ADS) und Sprachentwicklungsstörungen weder

Tabelle 5

Quecksilbermengen (Hg), die 6 Monate alte US-Säuglinge 1999 gemäss Impfplan erhielten, Vergleich mit EPA-Limiten.

\begin{tabular}{|c|c|c|c|c|}
\hline Impfstoff & Anzahl Dosen & $\mu \mathrm{g} \mathrm{Hg}$ pro Dosis Minimum & ug Hg pro Dosis Maximum & Berechnete Maximaldosen gemäss EPA-Limite \\
\hline DTaP & 3 & 0 & 75 & \\
\hline Hib & 3 & 0 & 75 & \\
\hline Hepatitis B & 3 & 0 & 37,5 & \\
\hline Influenza* & 1 & 12,5 & 12,5 & \\
\hline \multirow[t]{3}{*}{ Total } & & 12,5 & 200 (187,5 ohne Influenza) & $65 \mu \mathrm{g} \mathrm{Hg}$ für 5. Gewichtsperzentile \\
\hline & & & & $89 \mu \mathrm{g} \mathrm{Hg}$ für 50. Gewichtsperzentile \\
\hline & & & & $106 \mu \mathrm{g} \mathrm{Hg}$ für 95. Gewichtsperzentile \\
\hline
\end{tabular}


belegt, noch ausgeschlossen werden kann [26]. Das Komitee empfahl, thiomersalhaltige Impfstoffe nicht an Kinder zu verabreichen, da es die Hypothese eines Zusammenhangs zwischen thiomersalhaltigen Impfstoffen und der Entwicklung von neurologischen Störungen bei Kindern für biologisch plausibel hielt. Zum gleichen Schluss kam eine umfassende Literaturstudie von Bernard et al. [27], worin die Hypothese aufgestellt wurde, dass Autismus eine neue Form von Quecksilbervergiftung ist. Das Symptomenspektrum bei Autismus wurde mit demjenigen bei Quecksilbervergiftungen verglichen, und es zeigte sich eine starke Übereinstimmung. Im Mai 2004 publizierte das IOM erneut einen Bericht zum Thema Impfstoffe und Autismus [28]. Darin kamen die Experten zum Schluss, dass aufgrund neuster Daten kein kausaler $\mathrm{Zu}$ sammenhang zwischen Thiomersal in Impfstoffen und Autismus besteht. Diese Aussage basiert auf der Auswertung von fünf epidemiologischen Studien, welche seit 2001 in den USA, im UK, in Dänemark und Schweden durchgeführt wurden. Kohorten, die mit Impfstoffen höhere Mengen an Thiomersal erhielten, wiesen im Vergleich keine erhöhten Autismuszahlen auf. Auch die Europäische Zulassungsbehörde EMEA kam aufgrund einer eigenen Analyse zum Schluss, dass ein Zusammenhang zwischen Autismus und der Anwendung thiomersalhaltiger Impfstoffe nicht nachweisbar ist [29]. Diese Aussagen werden jedoch durch eine Studie der Columbia-Universität in New York relativiert. Mäuse, die aus genetischen Gründen besonders anfällig gegenüber Autoimmunerkrankungen sind, wurden Thiomersal ausgesetzt. Die Tiere zeigten bei Dosierungen, wie sie auch bei Impfungen für Kinder verwendet werden, Wachstumsstörungen, verminderte Beweglichkeit und eine übertriebene Antwort auf neue Reize. Im Hippokampus der Tiere fanden die Forscher dicht gepackte, hyperchrome Neuronen mit Veränderungen der Glutamatrezeptoren und der Glutamattransportmoleküle. Veränderungen im Neurotransmitterbereich sind typisch für Autismus. Bei Kontrolltieren ohne genetische Neigung zu Autoimmunreaktionen wurden keine Veränderungen beobachtet [30].

\section{Was sind mögliche Ursachen für die Entstehung von Autismus?}

Es gibt zahlreiche, stark divergierende Erklärungsansätze und Forschungsergebnisse darüber, was Autismus auslösen könnte. Nachfolgend sind einige erwähnt: a) Der autistischen Störung liegen hirnorganische Veränderungen zugrunde. Bei der Untersuchung autistischer Kinder sind in manchen Fällen gestörte Hirnwellenmuster und verminderte Hirndurchblutung festgestellt worden. Mit speziellen Methoden wie der Kernspintomographie wurden Veränderungen im Kleinhirn, Amygdala und Hippokampus entdeckt. Die Hirngebiete und -funktionen, bei denen eine Beeinträchtigung festgestellt wurde, stehen im Zusammenhang mit der Entwicklung von Sozialverhalten und Sprache. Das Ausmass der Hirnveränderungen scheint mit der Schwere der Symptomatik in Zusammenhang zu stehen. Besonders auffällig war die Dichteabnahme der Purkinjezellen im Kleinhirn, was zu einer Verkleinerung des Kleinhirns führt. Es wird davon ausgegangen, dass diese Schädigung pränatal auftritt. Kleinhirn und Hirnstamm bleiben im gesamten Entwicklungsverlauf kleiner als bei gesunden Personen. Bei 30\% der Erwachsenen, die in der Kindheit schwere autistische Symptome zeigten, treten epileptische Anfälle auf.

b) Als weitere Ursache für Autismus wird eine Veränderung im Neurotransmitterbereich angenommen, die bei der Untersuchung des Stoffwechsels von autistischen Menschen entdeckt wurde. Studien zeigen, dass bei 54\% der Autisten erhöhte Endorphinspiegel vorliegen. Charakteristisch ist dies besonders für Autisten, die zu Selbstverletzungen neigen und dabei kaum Schmerzempfindungen haben. Bei etwa der Hälfte der autistischen Kinder wird auf einen erhöhten Dopaminspiegel geschlossen. Dopamin beeinflusst hauptsächlich die Motorik, das Ess- und Trinkverhalten und die Kognition. Bei 25\% einer Gruppe von autistischen Kindern konnten erhöhte Serotoninspiegel beobachtet werden. Serotonin wirkt sich auf Schlaf, Essen, Schmerz, Stimmung oder auf Selbstund Fremdaggression aus. Die Störung des Haushalts dieser Botenstoffe wird zur Erklärung des problematischen Sozialverhaltens, der Aufmerksamkeitsdefizite und Lernschwierigkeiten autistischer Kinder herangezogen.

c) Autismus besitzt einen starken genetischen Hintergrund, erkennbar an einer familiären Häufung. Familien, die schon ein autistisches Kind haben, haben ein 60- bis 100fach höheres Risiko, noch einmal ein Kind mit frühkindlichem Autismus zur Welt zu bringen. Die Wahrscheinlichkeit, dass bei einem autistischen Zwillingskind auch der andere Zwilling betroffen ist, ist sehr hoch. Sie be- 
trägt bei eineiigen Zwillingen $60 \%$ und bei zweieiigen Zwillingen 5\%. In den 80er Jahren wurde festgestellt, dass bei einem autistischen Elternteil rund 50\% der Kinder ebenfalls von der Krankheit betroffen sind. Eine US-Studie untersuchte die Mitglieder von 411 betroffenen Familien und zeigte, dass zwei Varianten des Gens SLC25A12 auf Chromosom 2 besonders häufig vorkommen [31]. SLC25A12 kodiert für das Protein ACG1 (Aspartat/Glutamat-Carrier). ACG1 spielt eine wichtige Rolle im Aminosäurestoffwechsel der Mitochondrien und damit in der Energieversorgung der Zellen. Indem diese Genvarianten zu einer verstärkten Produktion des ACG1-Proteins führen, könnten sie die Funktion der energiehungrigen Nervenzellen beeinträchtigen. Mutationen auf den geschlechtsbestimmenden X- und Y-Chromosomen, die auch die Testosteronherstellung steuern, und beim Serotonintransportproteingen HTT, das für die Serotonintransportproteine kodiert, könnten möglicherweise auch eine wichtige Rolle spielen.

d) In der Autismusforschung hielt man lange Zeit den psychoanalytischen Aspekt für dominierend. Man ging davon aus, dass eine schwierige Eltern-Kind-Beziehung zur Ausbildung des autistischen Syndroms führt. Die Hirnveränderungen wurden als Folge der psychischen Verelendung («Kühlschrankmutter») erklärt. Diese Theorie wurde aber weitgehend verworfen. Zum einen konnte nicht nachgewiesen werden, dass Eltern autistischer Kinder besonders gefühlsarm und ablehnend sind, zum anderen treten die Symptome häufig schon im Säuglingsalter auf, was eher auf biologische Ursachen hinweist. Ungünstige soziale Bedingungen sind allenfalls krankheitsfördernde Faktoren, können aber für sich allein kaum so tiefgreifende Entwicklungsstörungen wie Autismus hervorrufen.

Weitere vermutete Ursachen sind: eine Rötelnerkrankung der Mutter während der Schwangerschaft [32] (Risiko für Autismus des Kindes 10mal höher als bei normaler Schwangerschaft), kongenitale Infektion mit Zytomegalovirus [33], Inutero-Kontakt mit Alkohol oder Medikamenten, Sauerstoffmangel während der Geburt, Erkrankungen des Kindes mit hohem Fieber, Umweltgifte, Ernährung, Zerstörung des Darmimmunsystems durch Antibiotika oder Infektionen mit Candida albicans. Eine Untersuchung des Plasmas autistischer Kinder zeigte signifikant erhöhte Autoantikörperspiegel gegen neuronale und gliale Filamentproteine, was auf eine Autoimmunerkrankung hinweist [34]. Kontrovers diskutiert als mögliche Ursache von Autismus werden neben den thiomersalhaltigen Impfstoffen auch die Impfungen gegen Masern, Mumps und Röteln (MMR). Hypothesen wurden aufgestellt, das die MMR-Impfung zu entzündlichen Veränderungen im Darm und damit zu einer erhöhten Durchlässigkeit der Darmschleimhaut für Umweltgifte führt [35]. Effektiv leiden viele autistische Kinder unter gastrointestinalen Beschwerden in Form von Durchfällen, Obstipation und Bauchschmerzen [36]. Ob die gastrointestinale Pathologie Teilursache oder eine mögliche Folge eines autistischen Leidens bei Kindern ist, bleibt offen. Eine Studie, durchgeführt vom englischen Entwicklungspsychologen Simon Baron-Cohen, postuliert, dass Kinder mit Autismus im Mutterleib erhöhten Testosteronspiegeln ausgesetzt waren. Das männliche Geschlechtshormon Testosteron beeinflusst auch die Entwicklung des Gehirns. In der Tat erinnert das Verhalten autistischer Patienten an männliche Klischees, wie ausgeprägtes Interesse an speziellen, meist technischen Themen wie z.B. Fahrpläne und Computer, Faszination für Details und mangelndes empathisches, soziales und kommunikatives Geschick. Den massiven Anstieg von Autismusfällen erklärt jedoch auch Baron-Cohens Testosterontheorie nicht.

Wahrscheinlich muss eine genetische Grunddisposition vorhanden sein, damit es zur Entwicklung von Autismus kommt. Zu einer Manifestation der Entwicklungsstörung könnte es schliesslich kommen, wenn äussere Faktoren, die bisher nicht bekannt sind, als Auslöser agieren. Ein solcher Stimulus könnte z.B. organisches Quecksilber, enthalten in Thiomersal aber auch in Zahnamalgam und Meeresfischen, sein. Daher sollten Kinder möglichst nicht mit diesen Substanzen in Kontakt kommen und Impfungen thiomersalfrei durchgeführt werden. Um Näheres dazu zu erfahren, wären Fallstudien sinnvoll, die Kinder mit regressivem Autismus bezüglich des Einflusses verschiedener Umweltfaktoren, wie auch bezüglich des Erhalts von thiomersalhaltigen und anderen Impfstoffen erfassen würden. Diese Kinder sollten dann bezüglich dieser Einflussfaktoren mit gesunden Kindern verglichen werden. Genetische Faktoren könnten ebenfalls für einen unterschiedlichen Metabolismus und verschieden rasche Elimination von Umweltgiften, wie z.B. Quecksilber, verantwortlich sein. Da Autismus über Verhaltensmodelle identifiziert wird, die beim Kind im Alter zwischen 18 Monaten und 3 Jahren deutlich werden, kann die Entwicklungsstörung 
noch nicht bei der Geburt diagnostiziert werden, obschon mögliche Ursachen bereits im prä- oder perinatalen Stadium liegen.

\section{Was ist 2-Phenoxyethanol und wie sicher ist die Substanz?}

2-Phenoxyethanol (2-PE, Abb. 2) ist ein aromatischer Etheralkohol, der hauptsächlich zur Konservierung von Kosmetika und topischen Arzneimitteln und als Fixativ in Parfüms in Konzentrationen von 0,5 bis $1 \%$ eingesetzt wird [37]. In Konzentrationen von 2\% wurde die Substanz auch therapeutisch, in Lösungen und Salben zur Desinfektion von oberflächlichen Wunden und Verbrennungen, eingesetzt [38]. 2-PE wird in Konzentrationen von 0,03\% zur Betäubung von Fischen verwendet. Andere Bezeichnungen für 2-PE sind: Arosol, Dowanol, Phenoxetol, Phenoxen, Uniphen P-23, Ethylenglykolmonophenylether oder Phenylcellosolve. Infolge der Inkompatibilität von Thiomersal mit inaktiviertem Poliomyelitisimpfstoff (IPV) [5, 6] musste nach einem alternativen Konservierungsmittel für diese Impfstoffe gesucht werden, das in 2-PE gefunden wurde [39-41]. 2-PE wird seit den frühen 80er Jahren in IPV-Impfstoffen zur Konservierung eingesetzt und ersetzt je länger je mehr Thiomersal als Konservierungsmittel in Kombinationsimpfstoffen (Tab. 2). 2-PE hat einen breiten antimikrobiellen Wirkungsbereich mit ausgeprägter Aktivität gegenüber gram-negativen Bakterien. Die grösste Wirksamkeit besitzt 2-PE gegenüber Pseudomonas aeruginosa (minimale Hemmkonzentration: 0,32\%). In höherer Konzentration wirkt 2-PE auch gegen gram-positive Bakterien (Staphylococcus aureus: 0,85\%) und Hefen (Candida albicans: 0,54\%). Oft wird 2-PE mit anderen Konservierungsmitteln (z.B. Parabenen) kombiniert. In Anwesenheit starker Säuren kann die Etherbindung gespalten werden, wobei Phenol und Ethanol entstehen. Der antimikrobielle Wirkmechanismus von 2-PE beruht u.a. auf einer Erhöhung der Zellmembrandurchlässigkeit für Kaliumionen [42, 43]. 2-PE ist in Spuren ein natürlicher Bestandteil von Grüntee [44].

\section{Abbildung 2}

Strukturformel von 2-Phenoxyethanol.

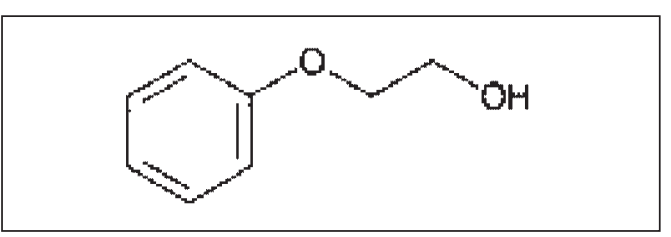

\section{Akute Toxizität}

Höhere Dosen von 2-PE sind schädlich nach Inhalation, peroraler Einnahme und Absorption über die Haut. Tierexperimentell wurden fruchtbarkeitsschädigende Wirkungen beobachtet [45]. Eine Entwicklungstoxizitätsstudie mit Süsswasserpolypen (Hydra attenuata) kam zum Schluss, dass 2-PE in Konzentrationen, die für Erwachsene noch unbedenklich sind, embryotoxisch ist [46]. Gemäss der EU-Richtlinie 91/155/EC ist 2-PE als schädlich nach Einnahme und reizend am Auge klassiert. Akute Toxizitätsdaten für 2-Phenoxyethanol sind in Tabelle 4 aufgeführt. Eine 10\%ige Lösung führt zu keiner Reizung der Kaninchenhaut und eine 2\%ige Lösung reizt das Kaninchenauge nicht [47]. Unverdünnt jedoch reizt 2-PE das Auge stark. 2-PE zeigte keine Mutagenität im Ames- und Maus-MikronukleusTest [48]. Die Konzentrationen, die zum Einsatz kamen, waren: 50, 150, 500, 1500 und $5000 \mu \mathrm{g}$ 2-PE pro Platte mit den Salmonella-typhimuriumStämmen TA 1535, TA 1537, TA 1538, TA 98 und TA 100. Im Maus-Mikronukleus-Test wurden Mengen von 300, 600 und 1200 mg 2-PE pro kg KG untersucht.

Nach Anwendung von 2-PE-haltigen, topischen Präparaten beim Menschen kommt es gelegentlich zu Kontaktallergien [49]. EpikutanTests (Patch-Tests) mit 2-PE, durchgeführt in England [50], den Niederlanden [51], in Italien [52] und Deutschland [53], zeigten sehr selten positive Resultate (unter 0,2\%). Ein Phototoxizitätstest, durchgeführt mit 2-PE an 28 Probanden im Alter zwischen 18 und 50 Jahren über eine Zeitspanne von 72 Stunden, fiel negativ aus [54]. In der Literatur sind nur vereinzelt Nebenwirkungen nach Gabe von 2-PE-haltigen Impfstoffen dokumentiert. So beschreiben Vogt et al. den Fall eines generalisierten Ekzems bei einem 18 Monate alten Jungen 24 Stunden nach der Verabreichung eines 2-PE-haltigen, kombinierten Diphtherie-, Tetanus- und Keuchhusten-Impfstoffes [55]. Die Dermatitis war stärker nach der zweiten Gabe des Impfstoffes und konnte mit topischen Kortikosteroiden behandelt werden. Der Hauttest für 2-PE war positiv. Die dritte Impfung zur Grundimmunisierung wurde dann mit einer thiomersalhaltigen Alternative durchgeführt, wobei es zu keiner allergischen Reaktion kam.

\section{Chronische Toxizität}

Von 113 norwegischen Medizinstudenten, die mit 1\%iger 2-PE-Lösung zur Konservierung von Leichen arbeiteten, zeigten $33 \%$ ungewöhnliche Müdigkeit und Schläfrigkeit und 27\% ungewöhnliche Kopfschmerzen. Die Symptome verschwanden, nachdem kein 2-PE mehr zur 
Konservierung verwendet wurde [56]. Aus der Publikation geht die Expositionsdauer nicht hervor. 1990 wurden drei Fälle von chronischer Neurotoxizität bei Personen publiziert, die mit 2-PE als Anästhetikum für Fische arbeiteten. Nach 1 bis 2 Jahren Exposition waren diese Personen infolge Wahrnehmungsstörungen, Vergesslichkeit, Konzentrationsschwäche, Schwindel, extremer Schläfrigkeit und Reizbarkeit nicht mehr arbeitsfähig. Es stellte sich heraus, dass die Arbeiter bis zu $500 \mathrm{~mL}$ 2-PE pro Tag verwendeten, das sie in Wasserbecken gossen, worin sie ohne Handschuhe Jungfische markierten. Die Aufnahme von 2-PE erfolgte perkutan. Als Sofortwirkung meldeten die Arbeiter Schwäche und Gefühllosigkeit der Hände und Kopfschmerzen. Die neurologischen Störungen blieben bestehen, nachdem die Exposition gestoppt wurde und glichen der ZNS-Toxizität von anderen organischen Lösungsmitteln [57]. 1999 publizierten Musshoff et al. Daten zur Wirkung von 17 Glykolethern auf die Glutamatrezeptorfunktion. Dabei resultierte für 2-PE eine deutliche Reduktion der N-Methyl-D-Aspartat (NMDA) induzierten Membranionenströme an Glutamatsubrezeptoren, die nach Injektion von Hirn-mRNA der Ratte in Xenopus-Oozyten exprimiert wurden. Die Reduktion der Membranionenströme war reversibel und dosisabhängig. Die restlichen 16 Glykolether zeigten keine solchen Effekte. Die Autoren schlossen auf ein starkes neurotoxisches Potential von 2-PE, was die Neurotoxizitätsfälle aus dem Jahre 1990 erklären würde [58]. Diese Arbeit wurde kritisiert und es wurde in Frage gestellt, ob die Hemmung des NMDA-Rezeptors durch 2-PE in Xenopus-Oozyten mit einem neurotoxischen Potential im Menschen gleichgesetzt werden darf $[59,60]$.

Es fällt auf, dass die chronische Toxizität von 2-PE in der Literatur nur lückenhaft untersucht und dokumentiert wurde. Namentlich ist wenig bekannt bezüglich Verteilung, Metabolisierung und Elimination nach parenteraler Gabe. Erfahrungswerte aus dem breiten und langjährigen Einsatz von 2-PE zur Konservierung von Kosmetika, topischen Arzneimitteln und parenteralen Impfstoffen ohne gravierende Nebenwirkungen

Abbildung 3

Strukturformel von Phenol.

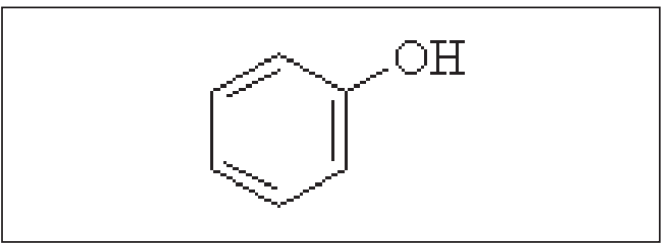

weisen nach dem aktuellen Stand des Wissens jedoch auf eine akzeptable Verträglichkeit von 2-PE hin.

\section{Was ist Phenol und wie sicher ist die Substanz?}

Phenol (Abb. 3) ist ein aromatischer Alkohol mit äusserst reaktiven und korrosiven Eigenschaften und starkem, charakteristischem Geruch. Die Substanz wurde ursprünglich zur Wunddesinfektion eingesetzt (erstmaliger Einsatz 1864 durch Lemaire in Frankreich und Lister in Schottland im Jahre 1867), jedoch infolge der hautirritierenden Wirkung bald durch andere Antiseptika ersetzt. Wegen der bakteriziden Wirkung war auch der Einsatz von Phenol als Desinfektionsmittel verbreitet. Gemäss Europäischer Pharmakopöe darf Phenol in Pharmazeutika in einer maximalen Konzentration von 2,5 mg/mL verwendet werden. Phenol war früher häufig in Sera und Impfstoffen (z.B. Pockenimpfstoff) zu finden und war u.a. auch bis in die 1980er Jahre Bestandteil der Solutio Castellani, die in der HNOMedizin bei Otitis externa eingesetzt wurde. Phenolhaltige Präparate werden trotz bekannt hoher Toxizität nach wie vor auch zur Faltenentfernung eingesetzt $[61,62]$. Tabelle 3 zeigt die von Swissmedic zugelassenen phenolhaltigen Impfstoffe mit Angabe der zugesetzten Menge. Andere Bezeichnungen für Phenol sind: Karbolsäure, Hydroxybenzol, Phenylsäure, Phensäure oder Phenylalkohol. Phenol ist antimikrobiell breit wirksam gegen gram-negative und grampositive Bakterien, Mykobakterien, einige Pilze und Viren. Phenol wirkt u.a. durch Denaturierung von Proteinen. Die antimikrobielle Wirksamkeit steigt mit zunehmender Temperatur und bei saurem $\mathrm{pH}$-Wert, ist jedoch gegenüber Sporen beschränkt. Wässrige, 1\%ige Lösungen sind bakteriostatisch, höher konzentrierte Lösungen dagegen bakterizid. Phenol sollte aus technischen Gründen nicht zur Konservierung von gefriergetrockneten Produkten eingesetzt werden und ist inkompatibel mit Albumin und Gelatine, die ausgefällt werden. Gemäss WHOAnforderung sind phenolische Komponenten nicht zugelassen zur Konservierung von Diphtherie- und Tetanus-Toxoid-Impfstoffen, da sie deren antigene Wirksamkeit negativ beeinflussen [63]. Phenol ist Bestandteil von Tabakrauch und wird physiologisch im menschlichen Körper produziert, z.B. im Rahmen des Proteinmetabolismus. Personen, die keinen Kontakt mit Phenol haben, scheiden die Substanz in Konzentrationen von 0,5 bis $80 \mathrm{mg} / \mathrm{L}$ Urin aus [64]. Gemäss 
der American Conference of Governmental Industrial Hygienists liegt dieser Wert bei Personen ohne Phenol- und Benzolkontakt nicht über $20 \mathrm{mg} / \mathrm{L}$ Urin und ist üblicherweise unter 10 mg/L Urin [65]. Ein Biomarker für Phenolexposition ist dunkler Urin.

\section{Akute Toxizität}

Die lokale toxische Wirkung von Phenol beruht auf der Denaturierung von Proteinen. Die Verätzungen sind nur anfänglich schmerzhaft, da Phenol lokalanästhetisch wirkt. Phenol wird über die intakte Haut aufgenommen, wobei die dermale Absorption von wässrigen Phenollösungen grösser ist als diejenige von reinem Phenol [66]. Die systemische toxische Wirkung beruht auf radikalvermittelten Reaktionen und manifestiert sich in Form von Arrhythmien, Lähmungen des ZNS mit Kollaps und starker Temperatursenkung, Leber- und Nierentoxizität. Das Einatmen von Dämpfen oder Nebel von Phenol führt zu starken Schädigungen der Atemwege. Nach dem Verschlucken wird die Schleimhaut von Mund, Rachen, Speiseröhre und Magendarmtrakt verätzt und es besteht Perforationsgefahr für Speiseröhre und Magen. Verätzungen am Auge können zum Erblinden führen. Nach Resorption kommt es zu Kopfschmerzen, Benommenheit, Rausch, Verwirrtheit, Bewusstlosigkeit, Herz-Kreislauf-Störungen, Blutbildveränderungen, Atemstillstand und Tod. Die maximale Arbeitsplatzkonzentration (MAK) von $5 \mathrm{~mL}$ respektive $19 \mathrm{mg}$ pro $\mathrm{m}^{3}$ Luft darf nicht überschritten werden. Die Geruchsschwelle von Phenol liegt bei 0,18 bis $22 \mathrm{mg} / \mathrm{m}^{3}$ Luft. Akute Toxizitätsdaten für Phenol sind in Tabelle 4 aufgeführt. Die tiefste publizierte tödliche Dosis beim Menschen ist $14 \mathrm{mg} / \mathrm{kg}$ peroral. Es gibt jedoch gemäss Literatur auch Individuen, die Dosen von 65 g Phenol (dies entspricht $930 \mathrm{mg} / \mathrm{kg}$ für einen $70 \mathrm{~kg}$ schweren Menschen) überlebt haben [67]. Die Durchsicht verschiedener Vergiftungsfälle durch Bruce et al. führte zu einer geschätzten minimalen oralen tödlichen Phenoldosis von $140 \mathrm{mg} / \mathrm{kg}$ [68].

\section{Aufnahme, Verteilung, Metabolisierung und Elimination}

Phenol wird zu 80-90\% über Atemwege, Haut und Gastrointestinaltrakt resorbiert. Die Substanz wird zur Entgiftung und Elimination überwiegend mit Sulfat oder Glukuronsäure konjugiert. Ein kleiner Teil wird zu Hydrochinon und Catechol oxidiert. Hydrochinon und Catechol werden ihrerseits konjugiert, aber auch ein weiterer Hydroxylierungsschritt zu 1,2,4-Trihydroxybenzol ist möglich. Konjugation mit Sulfat und Glukuronsäure erfolgt vorwiegend im Gastrointestinaltrakt, in Leber, Lunge und Nieren. Da die Haut diese Form der Entgiftung nicht vornehmen kann, ist Phenol insbesondere dermal sehr giftig. Sowohl Aufnahme, Verteilung wie auch Elimination erfolgen sehr rasch. Die Ausscheidung von Phenol und seinen Metaboliten erfolgt bei Mensch und Tier überwiegend mit dem Urin, wobei sich dieser aufgrund der Oxidationsprodukte von Phenol dunkel verfärbt. Bei Ratten liegt die Ausscheidung über den Urin bei 90-97\% und das Metabolitenprofil ist weitgehend unabhängig vom Applikationsweg. Die Halbwertzeit von Phenol beträgt im Menschen 3,5 Stunden. Phenolkonzentrationen im Urin von exponierten Personen können bis zu 200 mg/L Urin betragen [69].

\section{Chronische Toxizität}

Chronische Wirkungen von Phenol sind Appetitlosigkeit, Kopfschmerzen, Marasmus (Auszehrung, Entkräftung), Erschöpfung und chronische Schlafstörungen. In-vitro- und In-vivoMutagenitätsstudien lieferten in Abhängigkeit der untersuchten Spezies und eingesetzten Phenoldosis sowohl positive wie negative Resultate. Die Mutagenität von Phenol kann nicht abschliessend beurteilt werden, jedoch gibt es verschiedene Hinweise, dass Phenol in hohen Dosen mutagen sein kann.

\section{Vergiftungen}

Zahlreiche Fälle von Vergiftungen mit Phenol, mit tödlichem und ohne tödlichen Ausgang, sind publiziert. So beschreiben u.a. StajduharCaric einen tödlichen Fall nach Einnahme von 10 bis $20 \mathrm{~g}$ Phenol [70]. Der Tod bei dieser Frau trat innerhalb von Stunden mit Lungenödem und Atemstillstand ein. Griffiths beschreibt einen tödlichen Fall nach Hautkontakt mit flüssigem Phenol [71]. Dabei kamen 25\% der Körperoberfläche mit der Substanz in Berührung und der Tod trat innerhalb von 10 Minuten durch Herz- und Atemstillstand ein. Bei einem anderen tödlichen Fall nach Hautkontakt mit einer 80\%igen Phenollösung traten vorgängig Krämpfe auf [72]. Haddad et al. beschreiben einen Vergiftungsfall bei einer Frau, die versehentlich 26,7 g Phenol einnahm und den Vorfall überlebte [73]. Hingegen waren 24-26g Phenol peroral tödlich in einem von Lo Dico et al. beschriebenen Fall [74]. Horch et al. berichten von einem Mann, der sich eine wässrige Phenollösung unbekannter Konzentration auf Gesicht, Brust, Hände und Arme goss. Dabei traten neben Verbrennungen der Haut Arrhythmien und Bradykardie während 6 Stunden nach Exposition 
auf. Zusätzlich litt die vergiftete Person während einiger Tage unter Übelkeit und Erbrechen und die Serumbilirubinwerte waren um das Doppelte erhöht, wobei erst nach 5 Tagen die Normalwerte wieder erreicht waren [64].

\section{Zusammenfassung und Ausblick}

Ein Gefährdungspotential von Thiomersal bezüglich der Auslösung von Autismus bei empfindlichen Kindern kann gemäss heutigem Wissensstand nicht ausgeschlossen werden. Da jedoch heute in der Schweiz alle Kinderimpfungen, auch dank frühzeitiger und aktiver Intervention der Behörden, in thiomersalfreien Einzeldosen erhältlich sind und für Erwachsene, mit Ausnahme der Immunisierung gegen Leptospiren, thiomersalfreie Alternativen vorliegen, stellt Thiomersal in Impfstoffen kein akutes Problem für die Schweizer Bevölkerung dar. Swissmedic begrüsst und unterstützt eine Zukunft ohne Konservierungsmittel in Impfstoffmonodosen bei kontinuierlicher Verbesserung der Herstellprozesse. Dies gilt insbesondere für Impfungen der Grundimmunisierung, da diese bereits an 2 Monate alte Säuglinge verabreicht werden, deren Nerven- und Immunsystem in Entwicklung sind. Gemäss Auskunft von Impfstoffherstellern sind Bestrebungen im Gang, bei Monodosen gänzlich auf den Konservierungsmittelzusatz zu verzichten, wobei im Sinne einer NutzenSchaden-Analyse der Einhaltung der Sterilität gegenüber dem gänzlichen Verzicht auf ein Konservierungsmittel oberste Priorität zukommen soll. Oft geht mit dem Weglassen der Konservierungsmittel eine Einführung der Isolatortechnik zur aseptischen Abfüllung einher. Werden Impfantigene zwecks höherer Immunogenität mit Adjuvantien wie z.B. Aluminiumsalzen konjugiert bzw. daran adsorbiert, so erlaubt die Grösse der Moleküle keine Sterilfiltration mit Porengrösse $0,2 \mu \mathrm{m}$ bei der Abfüllung in die Endbehälter. Insbesondere in diesen Fällen und mit dem Trend, immer mehr Impfantigene in einer Spritze zu kombinieren, werden beim Weglassen von Konservierungsmitteln während der Herstellung und bei der Endformulierung hohe Anforderungen an eine aseptische Arbeitsweise während Transport, Lagerung und Abfüllung gestellt. Mehrfachdosen, wie sie insbesondere durch die WHO in Entwicklungsländern eingesetzt werden, müssen weiterhin konserviert werden.

\section{Danksagung}

Ich danke Urs Candrian, Daniel Häuptli, Thomas Hottiger und Urs Hess, Swissmedic, für das sorgfältige und kritische Durchsehen des Manuskripts.

Literatur

1 Wilson GS. The Hazards of Immunization. New York: The Athlone Press; 1967.

2 Bernier RH, Frank JA, Nolan TF. Abscesses complicating DTP vaccination. Am J Dis Child 1981; 135:826-8.

3 Simon PA, Chen RT, Elliot JA, Schwartz B. Outbreak of pyogenic abscesses after diphtheria and tetanus toxoids and pertussis vaccine. Pediatr Infect Dis J 1993;12:368-71.

4 Schweizerischer Impfplan 2005. Supplementum VIII, Ordner «Infektionskrankheiten - Diagnose und Bekämpfung». Stand: Januar 2005. Bundesamt für Gesundheit und Eidgenössische Kommission für Impffragen.

5 Davisson EO, Powell HM, MacFarlane JO, Hodgson R, Stone RL, Culbertson CG. The preservation of poliomyelitis vaccine with stabilized methiolate. J Lab Clin Med 1956;47:8-19.

6 Sawyer LA, McInnis J, Patel A, Horne AD, Albrecht P. Deleterious effect of thimerosal on the potency of inactivated poliovirus vaccine. Vaccine 1994;12:851-6.

7 Cox NH, Forsyth A. Thiomersal allergy and vaccination reactions. Contact Dermatitis 1988; 18:229-33.

8 Goncalo M, Figueiredo A, Goncalo S. Hypersensitivity to thimerosal: the sensitivity moiety. Contact Dermatitis 1996;34:201-3.

9 Powell HM, Jamieson WA. Merthiolate as a germicide. Am J Hyg 1931;13:296-310.

10 Axton JMH. Six cases of poisoning after a parenteral organic mercurial compound (merthiolate). Postgrad Med J 1972;48:417-21.

11 Rohyans J, Walson PD, Wood GA, Mac Donald WA. Mercury toxicity following merthiolate ear irrigations. J Pediatr 1984;104:311-3.

12 Pichichero ME, Cernichiari E, Lopreiato J, Treanor J. Mercury concentrations and metabolism in infants receiving vaccines containing thiomersal: a descriptive study. Lancet 2002; 360:1737-41.

13 Oskarsson A, Schuetz A, Skefving S, Hallen IP, Ohlin B, Lagerkvist BJ. Total and inorganic mercury in breast milk and blood in relation to fish consumption and amalgam fillings in lactating women. Arch Environm Health 1996;51:234-41.

14 Bigham M, Copes R, Srour L. Exposure to thiomersal in vaccines used in Canadian infant immunization programs, with respect to risk of neurodevelopmental disorders. Can Commun Dis Rep 2002;28:69-80. 
15 Mahaffey KR, Rice G, Swartout J. An Assessment of Exposure to Mercury in the United States: Mercury Study Report to Congress (Document EPA-452/R097-006). Washington, DC: US Environmental Protection Agency; 1997.

16 World Health Organization. Trace Elements and Human Nutrition and Health. Geneva: WHO; 1996.

17 EMEA. Public Statement on Thiomersal Containing Medicinal Products (Doc Ref EMEA/20962/ 99). London: European Agency for the Evaluation of Medicinal Products; 8 July 1999.

18 EMEA. Position Statement on Recent Developments Concerning Thiomersal in Vaccines (Doc Ref EMEA/CPMP/1578/00). London: European Agency for the Evaluation of Medicinal Products; 29 June 2000.

19 Notice to Readers: Thiomersal in Vaccines: a Joint Statement of the American Academy of Pediatrics and the Public Health Service. MMWR 1999; 48:563-5.

20 EMEA. Points to Consider on the Reduction, Elimination or Substitution of Thiomersal in Vaccines (Doc Ref CPMP/BWP/2517/00). London: European Agency for the Evaluation of Medicinal Products; 26 April 2001.

21 Center for Biologics Evaluation and Research. Letters to manufacturers regarding plans for continued use of thimerosal as a vaccine preservative. July 1, 1999 and May 31, 2000.

22 Harada M. Minimata disease: methylmercury poisoning in Japan caused by environmental pollution. Crit Rev Toxicol 1995;25:1-24.

23 Bakir F, Damlugi SF, Amin-Zaki L, Murtadha M, Khalidi A, Al-Rawi NY, et al. Methylmercury poisoning in Iraq. Science 1973;181:230-41.

24 California Department of Developmental Services. Autistic Spectrum Disorders: Changes in the California Caseload. An Update: 1999 Through 2002.

25 Ball LK, Ball R, Pratt RD. An assessment of thiomersal use in childhood vaccines. Pediatrics 2001;107:1147-54.

26 Stratton K, Gable A, McCormick MC (eds.). Immunization Safety Review: ThiomersalContaining Vaccines and Neurodevelopmental Disorders. Washington, DC: National Academy Press; 2001.

27 Bernard S, Enayati A, Redwood L, Roger $\mathrm{H}$ Binstock T. Autism: a novel form of mercury poisoning. Med Hypotheses 2001;56:462-71.

28 Stratton K, Gable A, McCormick MC (eds.). Immunization Safety Review: Vaccines and Autism. Washington, DC: National Academy Press; 2004.

29 EMEA. Public Statement on Thiomersal in Vaccines for Human Use - Recent Evidence Supports Safety of Thiomersal Containing Vaccines (Doc Ref EMEA/CPMP/VEG/1194/04/Adopted). London: European Agency for the Evaluation of Medicinal Products; 24 March 2004.

30 Hornig M, Chian D, Lipkin WI. Neurotoxic effects of postnatal thimerosal are mouse strain dependent. Mol Psychiatry 2004;9(9):833-45.
31 Ramoz N, Reichert JG, Smith CJ, Silverman JM, Bespalova IN, Davis KL, et al. Linkage and association of the mitochondrial aspartate/ glutamate carrier SLC25A12 gene with autism. Am J Psychiatry 2004;161:662-9.

32 Chess S, Fernandez P, Korn S. Behavioral consequences of congenital rubella. J Pediatr 1978;93:699-703.

33 Ivarsson SA, Bjerre L, Vegfors P, Ahlfors K. Autism as one of several abnormalities in two children with congenital cytomegalovirus infection. Neuropediatrics 1990;21:102-3.

34 Singh VK, Warren R, Averett R, Ghaziuddin M. Circulating autoantibodies to neuronal and glial filament proteins in autism. Pediatr Neurol 1997;17:88-90.

35 Wakefield AJ, Murch SH, Anthony A, Linnell J, Casson DM, Malik M, et al. Ileal-lymphoid-nodular hyperplasia, non-specific colitis, and pervasive developmental disorder in children. Lancet 1998;351:637-41.

36 Melmed RD, Schneider CK, Fabes RA, Philipps J, Reichelt K. Metabolic markers and gastrointestinal symptoms in children with autism and related disorders. JPGN 2000;31:S2,A116.

37 Poudrier JK. Final Report on the Safety Assessment of Phenoxyethanol. J Am Coll Toxicol 1990;9(2):259-77.

38 Lawrence JC, Cason JS, Kidson A. Evaluation of phenoxethol-chlorhexidine cream as a prophylactic antibacterial agent in burns. Lancet 1982; 1:1037-40.

39 Pivnick H, Tracy JM, Tosoni AL, Glass DG. Preservatives for poliomyelitis (Salk) vaccine III: 2-phenoxyethanol. J Pharm Sci 1964;53:899-901.

40 Hekkens FEN, Polak-Vogelzang AA, Kreeftenberg JG The antimicrobial effectiveness of some preservatives in inactivated human vaccines. J Biol Stand 1981;9(3):277-85

41 Lowe I, Southern J. The antimicrobial activity of phenoxyethanol in vaccines. Lett Appl Microbiol 1994;18(2):115-6.

42 Gilbert P, Beveridge EG, Crone PB. The lethal action of 2-phenoxyethanol and its analogues upon Escherichia coli NCTC 5933. Microbios 1977; 19:125-41.

43 Beveridge EG, Boyd I, Jessen GW. The action of 2-phenoxyethanol upon Pseudomonas aeruginosa NCTC 6749. J Pharm Pharmacol 1980;32:17P.

44 Yamaguchi K, Shibamoto T. Volatile constituents of green tea, Gyokuro (Camellia sinensis L. var Yabukita). J Agric Food Chem 1981;29:366-70.

45 National Toxicology Program. Final Report Ethylene glycol monophenyl ether: Reproduction and fertility assessment in CD-1 mice when administered in feed. National Institute of Environmental Health Sciences. Pub. No. NTP-84-410.

46 Johnson EM, Gabel BE, Larson J. Developmental toxicity and structure/activity correlates of glycols and glycol ethers. Environ Health Perspect 1984; 57:135-9.

47 Nipa Laboratories Ltd. Technical literature: Phenoxetol, 1992. 
48 Huntingdon Research Centre. Ames metabolic activation test assess the potential mutagenic effect of phenoxetol. Micronucleus test on Phenoxyethanol. 1988 (unpublished data).

49 Bohn S, Bircher AJ. Phenoxyethanol-induced urticaria. Allergy 2001;56(9):922-3.

50 Lovell CR, White IR, Boyle J. Contact dermatitis from Phenoxyethanol in aqueous cream BP. Contact Dermatitis 1984;11(3):187.

51 DeGroot AC, Bos JD, Jagtman BA, Bruynzeel DP, VanJoost T, Weyland JW. Contact allergy to preservatives II. Contact Dermatitis 1986;15(4): 218-22.

52 Tosti A, Vincenzi C, Trevisi P, Guerra L. Euxyl K 400: incidence of sensitization, patch test concentration and vehicle. Contact Dermatitis 1995; 33:193-5.

53 Fuchs T, Estenders F, Przybilla B. Contact allergy to Euxyl K 400. Dermatosen 1991;39:151-3.

54 Hill Top Research Inc. Phototoxicity study, March 12, 1984. Report no. 83-0973-70 (unpublished data).

55 Vogt TH, Landthaler M, Stolz W. Generalized eczema in an 18-month-old boy due to Phenoxyethanol in DPT vaccine. Contact Dermatitis 1998; 38:50-1.

56 Frolich KW, Andersen LM, Knutsen A, Flood PR. Phenoxyethanol as a non-toxic substitute for formaldehyde in long-term preservation of human anatomical specimens for dissection and demonstration purposes. Anat Rec 1984; 208:271-8.

57 Morton WE. Occupational phenoxyethanol neurotoxicity: a report of three cases. J Occup Med 1990;32(1):42-5

58 Musshoff U, Madeja M, Binding N, Witting U, Speckmann EJ. Effects of 2-phenoxyethanol on N-methyl-D-aspartate (NMDA) receptor-mediated ion currents. Arch Toxicol 1999;73(1):55-9.

59 Schmuck G, Steffens W, Bomhard E. 2-phenoxyethanol: a neurotoxicant? Arch Toxicol 2000; 74:281-3.

60 Musshoff U, Madeja M, Binding N, Witting U, Speckmann EJ. 2-phenoxyethanol: a neurotoxicant? Reply. Arch Toxicol 2000;74:284-7.
61 Baker TJ, et al. Chemical face peeling for facial wrinkling. JAMA 1974;228:898-9.

62 Ariagno RP, Briggs DR. Chemexfoliation as an adjunct to facial rejuvenation. Trans Am Acad Ophthalmol Otolaryngol 1975;80:536-9.

63 WHO Expert Committee on Biological Standardization. Requirements for diphtheria, tetanus, pertussis, and combined vaccines. WHO Tech Rep Ser 800, 1990.

64 Horch R, Spilker G, Stark GB. Phenol burns and intoxications. Burns 1994;20(1):45-50.

65 ACGIH Threshold limit values for chemical substances and physical agents and biological exposure indices. American Conference of Governmental Industrial Hygienists. Cincinnati, OH. 1998;55:103.

66 Conning DM, Hayes MJ. The dermal toxicity of phenol: an investigation of the most effective first-aid measures. Br J Ind Med 1970;27:155-9.

67 Deichmann WB, Keplinger ML. Phenols and phenolic compounds. In: Clayton GD, Clayton FE (eds.). Patty's industrial hygiene and toxicology. $3^{\text {rd }}$ edition. New York, NY: John Wiley and Sons, Inc; 1981. p. 2567-627.

68 Bruce RM, Santodonato J, Neal MW. Summary review of the health effects associated with phenol. Toxicol Ind Health 1987;3:535-68.

69 Tesarova E, Packova V. Gas and high performance liquid chromatography of phenols. Chromatographia 1983;17:269-84

70 Stajduhar-Caric Z. Acute Phenol Poisoning. Singular findings in a lethal case. J Forensic Med $1968 ; 15: 41-2$

71 Griffiths GJ. Fatal acute poisoning by intradermal absorption of phenol. Med Sci Law 1973;13:46-8.

72 Lewin JF, Cleary WT. An accidental death caused by the absorption of phenol through skin. A case report. Forensic Sci Int 1982;19:177-9.

73 Haddad LM, Dimond KA, Schweistris JE. Phenol poisoning. JACEP 1979;8(7):267-9.

74 Lo Dico C, Caplan YH, Levine B, Smyth DF, Smialek JE. Phenol: Tissue distribution in a fatality. J Forensic Sci 1989;34:1013-5. 